\title{
A PROPOSAL FOR COORDINATOR CONTROL RECIPE IN A BATCH PROCESS
}

José Francisco Briones de la Torre

Polytechnic University of Aguascalientes

Av. Prol. Mahatma Gandhi km. 2

20280 Aguascalientes, MEXICO
Antonio Espuña Camarasa

Polytechnic University of Catalonia

ETSEIB, Av. Diagonal 647

08028 Barcelona, SPAIN

Luis Puigjaner i Corbella

Polytechnic University of Catalonia

ETSEIB, Av. Diagonal 647

08028 Barcelona, SPAIN

\begin{abstract}
In this work, we propose a coordinator control recipe in the context of a batch process with the use of elements of petri nets and some techniques associated with non linear control (e.g. the relative degree) focused to explain the implications of use a hybrid dynamical models in terms of problem control and its relation with on-line experimental measures and the norm ISA S88.02
\end{abstract}

\section{INTRODUCTION}

The manufacture of services and products is oriented to satisfy the exigencies of the consumers in terms of quality and accessibly of it and in a productive process point of view it is necessary to consider the comparative between the costs of production and commercialization. Both elements are important to define the economic viability of the enterprise (Staff 1998).

The enterprise must generate conditions to optimize the production of services and products (i.e. reduction of costs and time involved in its manufacture) and satisfies the necessities of the consumers with the consideration of such necessities become more diversities and complexes. A one way to configure the operations of a production process is using batch process philosophy as an option to achieve the proposed conditions (Carreira 2004, Simensen and Foss 1996).

The importance of batch processes is the advantage that they have in terms of avoid bottleneck in the process and the death time in units of production, additionally the batch process has the characteristic to adapt its configuration to respond the requirements of the final consumer in real time (Petrick and Culbert 1998). Then its necessary to have a production's methodology that balance the final consumers' necessities and the capacity of the enterprise to satisfy that necessities using an effective decision making applied at the enterprise's process improvements and changes in the configuration of the productive process like for example the recirculation of some kind production lines and divide the set of production task among the set of production units.

The ISA S88 family norms had been created as an answer to support the production' s methodology described in the previous paragraph and the decision making can be translated as control specifications, all of them applied at the typical components of production process as: (i) final consumer' s requirements; (ii) the specifications of raw materials; (iii) the operation's conditions associated with the use of specify units, the time occupied to complete a specific production task, the logistic of production, the specifications of the final product, the control variables to management the process, the schedule of production, the process operations to develop in the units of production, etc.; (iv) the control strategies to implement in the process from a specific context for example the P, PI, PID controls used in the control recipe to a global context associated with another kind of complex control strategies as control expert and adaptable control contained in the coordinator control recipe. Both recipes are applied at the set of interested control variables with the objective to obtain the final product derivate from process production in the specifications that satisfies the consumers' necessities (Santos 2001).

In this paper its propose at certain control strategy focused at the ISA S88 norm and adapted at a batch process in terms of the coordination control recipe and try to solve the typical control problem (i.e regulation and servo-control) considering all the elements applied at the dynamical analysis of feedback process: (i) the control reference proposed as the production' s schedule; (ii) the perturbations generated by the uncertainties, and nature of dynamical events (i.e. discrete of continuous) derivate from the modification in the different levels of batch process' operation and pointed 
out by the ISA S88 norms; (iii) the interconnections used in the batch process that it could be simple or complex with a variety of numbers of its like an option to control the process and achieve the reference (i.e. the schedule production) and solve the typical control problem .

\section{METHODOLOGY APPLIED TO DESIGN THE CONTROL RECIPE}

A batch process can be described by state models that provide relations among process variables and the ISA S88 breaks production into two key categories. One is procedural, which says how a product is made (the recipe). The other is equipment models that describe equipment and how it operates. Within the processes are the capabilities of each unit. These state models have many layers. One of this layers is the operations made in a unit of production which it can be described for control objectives as continuous dynamic system. Thus, for example if the unit contains a reactive mixture of reactants (i.e. continuous reactor stirred tank) and each reactant consumed and product formed have initial conditions and the reaction process has been developed until its reach a stationary equilibrium condition when the variable of the process (i.e. reactant and product concentrations) has been achieve a stationary equilibrium value. This part of the batch process had been modeled with the use of engineering' $\mathrm{s}$ basic principles by sets of partial differential equations:

$$
\frac{\partial \varphi}{\partial t}+(\nabla \cdot \varphi v)+(\nabla \cdot \varphi)-\dot{\varphi}_{g}=0
$$

Where in the set of equations 1 the $\varphi$ represents a process variable of interest (e.g. the consumption of a reactant and the formation of a product) and its describes the nature of the process (i.e. the kind of reaction where is developed in the unit); the time partial derivate describes the dynamical behavior of the process variable meanwhile the terms associated whit the gradient describe the evolution of the process variable in terms of dimensional variables and finally the - element describes the generation and/or consumption of the process variable. But the set of equations 1 has been applied a different kind of engineering processes (i.e. chemical, mechanical, electrical, electronic process) because the system 1 has been created using engineering' $\mathrm{s}$ basic principles and with the use of certain supposition obtained from the nature of the process, the system 1 is represented by a set of ordinary differential equations:

$$
\frac{d \varphi}{d t}=\frac{F}{V}\left(\varphi_{0}-\varphi\right)+\eta(\varphi, t)
$$

The set of equation 2 is an integral approach of the system 1 when the unit of production is consider as a control volume with multiple input (e.g. the set of reactants) and multiple output (e.g. the set of products). The multiple output from a specific unit can be connected at other unit or units and in this way establish the process relationship as transitions among the units and the reactants and products will became the tokens for the Petri net. Thus, in $2 F$ describes the flux of the process variable in a control volume, $V$ is the control volume related with the unit's total volume, $\varphi$ is the process variable, the subindex 0 represents the initial condition of the process variable at time $t=0$ and the $\eta(\varphi, t)$ is related with the generation and/or consumption of the process variable referring at the control volume.

The principal complication of the system 2 is the term $\eta(\varphi, t)$ because it is involved with system's uncertainties derivate from the number and type of suppositions used to convert the system 1 to 2 . Thus the term $\eta(\varphi, t)$ can be numerically constructed with process' measures from the process variable $\varphi$ because it is consider available to be measured and it is consider as the token for the petri net. The advantage to construct the term $\eta(\varphi, t)$ is robust the 2 dynamical system because all the uncertainties related with it can be measured and use this measure in fault diagnosis and improve the control strategies in terms of it can be physically feasible.

The condition for the control strategy of physically feasible is derivate by the schedule of production (where there are contained all the product specifications and the consumer necessities to be covered by the final product) and the physically feasible is defined as the possibility to achieve the reference with all the implications involved in the problem control itself. Then the system 2 can be designed using the ideas reported in (Teel and Praly 1995):

$$
\begin{aligned}
& \frac{d \hat{\varphi}}{d t}=\frac{F}{V}\left(\varphi_{0}-\hat{\varphi}\right)+\hat{\eta}(\hat{\varphi}, t)+L_{1}(\varphi-\hat{\varphi}) \\
& \frac{d \hat{\eta}}{d t}=L_{2}(\varphi-\hat{\varphi})
\end{aligned}
$$

Where in the system 3 the variables with the element $\wedge$ determinate the robust estimated variables such as under certain conditions the observation gains $L_{1}$ and $L_{2}$ with the error estimation $(\varphi-\hat{\varphi})$ the system 3 is equivalent at the system 2 with the advantage provided by the agglutination of uncertainties.

In this way the Petri nets use robust tokens to describe the discrete dynamical behavior of the process (derivate from the multiple inputs and multiple outputs among units of production), thus the system 3 will converge to 2 in the numerical description of the process variable $\varphi$ at a certain $\hat{\varphi}$ when the time $t$ tends to a constant value $\varphi *$ witch will be the transition among the production units and the Petri net activation.

The Petri net activation with the coordinator control recipe can be configure from the definition of the batch 


\section{Briones, Espuña, and Puigjaner}

process $(B P)$ by (Barros and Perkusich 1998):

$$
B P=(U B R)
$$

Where $U$ is a non-empty set of process variables $(\varphi)$ contained in the units that belongs to the batch process. $B$ is the non-empty set of procedures executed in each unit of production. $R$ is the non-empty set of recipes executed by all the batch process (i.e. the recipes that describe the how the product is made). The system 4 expressed in terms of Petri net $(P N)$ is:

$$
P N=\left(P, T ; F, K, W, M_{0}\right)
$$

In the system 5 the variable $P$ is the set of available arrive places for the tokens in the net, i.e. the quantity of multiple inputs related with the unit. $T$ is the set of transitions among places and physically identified by the number of transitions related with the units of production, i.e. the interconnection between the multiple outputs from a specific unit with the multiple inputs for a specific unit. $F$ is the set of interconnections establish among the units of productions, i.e. the relation of circulation among units defined to optimize the batch process and the main object to control by the control strategy defined by the coordinator control recipe. $K$ is the capacity function that describes the quantity of tokens for every arrive place $p \in\{P\}$, i.e. the quantity of reactive that can be handled by the reactor. $W$ is the weight function that defines the quantity of tokens available to circle through the interconnections $f \in\{F\}$, i.e. the quantity of reactants and products fluxes that circulate through the pipes that interconnects the units. $M_{0}$ is the token' $\mathrm{s}$ departure function, i.e. the initial condition for a certain reactive involved at the unit.

The system 4 is related with the system 5 through to consider the set $U$ defines the set $P$, the set $B$ defines the sets $K$ and $W$, the set $R$ defines the sets $T F$ and $W$, finally $M_{0}$ is the condition of the process variable $\varphi$ when its reaches the value of $\varphi *$.

\section{RESULTS}

The system 5 is a discrete dynamical system with elements obtained from a continuous dynamical system because is connected to the system 3 by the process variable $\varphi$. The cited connections become from the consideration of a set of outputs from a unit of production ( $\varphi$ as a product) can be the set of inputs for a unit of production ( $\varphi$ as a reactant), but the interaction between the both sets is not immediately because is necessary to complete a certain time of reaction and depends of this quantity of time to fix the discrete time interval associated in the transition between units of production.
Previously in this paper has been cited that the recirculation among units of production is a way to improve the performance of the batch process even thought the control strategies commonly use the feedback as a regular form to manipulate the plant, then exist the necessity to identify if the recirculation or the feedback relationship established in the process are the sufficient to guarantee the process optimization and the physical feasibility of the control strategies. Thus in a discrete system developed in a batch process exist a proposal refers from the system 5 can be obtained a incidence matrix of tokens defined by $A$ with components $a_{i j}=a_{i j}^{+}-a_{i j}^{-}$where the $a_{i j}$ elements are the transitions in the Petri net from the $i$ to $j$ places in certain direction ( + ) and a countercurrent direction $(-)$ to describe the variable process flux to establish equation:

$$
M_{k}=M_{k-1}+A^{T} u_{k}
$$

The equation 6 provides the evolution of the tokens in the Petri net through describes the $M_{k}$ position or unit of process related with a previous unit of process (that is the $M_{k-1}$ position) and the additional term is the evolution of the tokens (described by the $A$ matrix) and its relation with the elements of the $u_{k}$ vector. With the last definition has been projected a mathematical tool to describe the way in which the recirculation among unit of production focused to optimize the process and the forms to establish the control feedback can be feasible.

From the system 6 under the consideration of the set $M$ with the $A$ matrix are time invariants, the derivate in time of the system 6 allow the existence for a set of ordinary differential equations that describe in a continuous dynamical form the behavior of the Petri net. This approach applied to describe a discrete dynamical behavior with the use of continuous dynamical ordinary differential equations help to define the next: Due to the system 6 is related with the continuous dynamical behavior of the Petri net defined for $d u / d t$ with the number of interconnections established by the units of production $(F)$ the relation between it is provided by the system relative degree obtained with the use of Lie algebra:

$$
\begin{aligned}
& L_{u}^{0} F(m)=F(m) \neq 0 \\
& L_{u}^{k} F(m)=L_{u}\left(L_{u}^{k-1} F(m)\right)(m)=0
\end{aligned}
$$

Where the $L$ operator is the Lie derivate and the subindex defines the mathematical projection (in this case the vectorial field $u$ ) in the set of interest given by $F$ as $F$ is the set of interconnections among units of production $m \in\{M\}$ defined by the quantity of tokens to circulate in the Petri net. The term $k-1$ is the system relative degree. The system 7 is developed until the $k$ derivate is equal to zero and when its happens, by definition (Isidori 1989), the system relative degree is equal to $k-1$. 
Thus in the proposal context the relative degree means the interconnection established in the Petri net is equal to the number of tokens contained in the Petri net and if does not achieve then it is necessary to consider the presence of non-modeled dynamics and in the context of the Petri nets it means that certain connections had not been used or the number is not enough for the numbers of tokens in the Petri nets.

With this information exists the possibly to identify the options that the coordinator control recipe has to manipulate the process with feedback actions and the options to fix the recirculation relationships (interconnections among units of production) that added to the coordinator control recipe provide to optimization and a robust behavior at the global batch process.

\section{CONCLUSIONS}

By the definition of the system relative degree applied a the continuous dynamical description of the Petri net allow to define a structure of coordinator control recipe for a batch process and by the information obtained with the relative degree, the design of the Petri net can be optimize with the exact definition of the transitions among the units of production and the set of recipes of the process with it can propose a solution for the control problem related with this kind of process.

\section{REFERENCES}

Barros, C., and A. Perkusich. 1998. Simulation of the continuos dynamics of batch control systems based on petri nets. IEEE International Conference on Systems, Man, and Cybernetics 1:680-685.

Carreira, B. 2004. Lean manufacturing that works: Powerful tools for dramatically reducing waste and maximizing profits. USA: AMACOM.

Isidori, A. 1989. Nonlinear control systems: An introduction. Germany: Springer - Verlag.

Petrick, M., and S. Culbert. 1998. Automation philosophy of a hybrid plant. IEEE International Symposium on Industrial Electronics 2:628-633.

Santos, E. 2001. Understanding s88 batch control. $A-B$ Journal 8(5):100-105.

Simensen, J., and B. Foss. 1996. A concept for modeling batch plan operation. Computers and Chemical Engineering 20:S1245-S1250.

Staff, N. R. C. 1998. Manufacturing, process controls for the industries of the future. USA: National Academies Press.

Teel, A., and L. Praly. 1995. Tools for semiglobal stabilization by partial state and output feedback. SIAM Journal on Control and Optimization 33:1443-1488.

\section{AUTHOR BIOGRAPHIES}

JOSE FRANCISCO BRIONES DE LA TORRE is a Researcher Professor of Chemical Engineering in the Polytechnic University of Aguascalientes. He is developing his doctoral formation in the Center for Process and Environment Engineering in the Polytechnic University of Catalonia. He participates with other groups of research as the Supply Chian Academic Body in the Polytechnic University of Aguascalientes and in the same Institution he is the Head of Academic Development and Research since May 2007 and before it he was the Head of Mechanical Engineering and Head of Mechatronic Engineering Departments. His email address for these proceedings is <francisco.briones@upa.edu.mx>.

ANTONIO ESPUÑA CAMARASA is Professor of Chemical Engineering at Polytechnic University of Catalonya and Group Manager in the Center for Process and Environment Engineering. His research interests are mainly process modelling for integration and control, and also planning, scheduling and control of batch processes, with special emphasis on the use of artificial intelligence tools on these topics. Since 1985, he has participated in more than 20 RTD projects in these areas, both financed by private industries and organizations (Courtaulds, IBM, Bayer, etc.) and public research institutions (E.U., through ESPRIT and JOULE programs, Spanish Ministry of Science, Catalan Interdepartamental Commission for Research and Technological Innovation-CIRIT, etc.). He has published over 40 research papers on these topics and contributed in more than 80 national and international scientific meetings.

LUIS PUIGJANER I CORBELLA is Professor of Chemical Engineering at Polytechnic University of Catalonya and Director of the Environmental Center (LCMA) a non-profit agency for environment audit and engineering services. He is the Head of the research team on Design, Operation and Control of Chemical and Biotechnology Processes, which involves 35 researchers. His research interests are mainly concerned with process simulation and optimization and with the development of AI supporting techniques and software. He has supervised $33 \mathrm{PhD}$ Thesis in those areas. $\mathrm{He}$ has published over 300 papers in scientific journals and conference proceedings and the author of over 100 technical reports and 8 books. 